\title{
AOR
}

Selected Papers of \#AolR2019:

The $20^{\text {th }}$ Annual Conference of the Association of Internet Researchers Brisbane, Australia / 2-5 October 2019

\section{DON'T JUST SAY THANK YOU: EXPLORATION OF TYPES OF POSTS INSPIRING AND HINDERING DEEP CONVERSATIONS ONLINE.}

\author{
Devayani Tirthali \\ Independent Researcher \\ Yumiko Murai \\ Massachusetts Institute of Technology
}

How can we create an online learning environment that is so engaging that learners keep learning together beyond the given course period? This paper reports on an experience of a free open online course "Learning Creative Learning" launched in the fall of 2017. Primary aim of the course was to facilitate the development of a community of educators and designers as they learned about the creative learning framework through engagement in hands-on, peer-supported, project-based activities (Resnick, 2017).

Cormier (2014) experimented with the idea of 'community as the curriculum' in his open online course on rhizomatic education. His objective was to "organize an ecosystem where people form affinity connections" that continue beyond the course. Another example is Siemen and Downes' cMOOC on Connectivist learning created with a vision of learners owning their interaction space (Siemens in Hollands \& Tirthali, 2014). Both these courses were spread out across various platforms chosen by the participants instead of a central course space and did not follow a pre-set curriculum. Many participants thrive with the openness but some also flounder due to the lack of structure (Mackness \& Bell, 2015).

The course Learning Creative Learning tried to find a space in between the highly structured instructor-centred space of a Massive Open Online Course (MOOC) and the wide open space of the cMOOCs. It was organized as self-paced modules including a short video, reading materials, a hands-on activity, reflection prompts, and extra reading

Suggested Citation (APA): Tirthali, D. \& Murai Y. (2019, October 2-5). Don't just say thank you: Exploration of types of posts inspiring and hindering deep conversations online. Paper presented at AoIR 2019: The $20^{\text {th }}$ Annual Conference of the Association of Internet Researchers. Brisbane, Australia: AoIR. Retrieved from http://spir.aoir.org. 
materials for those interested in learning more. In addition to the facilitator prompted structured discussions every week, an open discussion forum was created where participants could start their own discussion thread. By creating this open online discussion space, the course attempted to create an environment similar to participantdriven open online communities.

In contrast to most MOOCs, that are often highly structured and instructor-driven (Swan, Day, Bogle, \& Prooyen, 2014), participant-driven open online communities, where people gather to share questions and seek information related to their interest on the platforms such as Twitter, Reddit, or Stack Overflow, tends to achieve high and sustained engagement. This is because open online environments tend to provide freedom for the participants to discuss topics they are most interested in, with people they are interested to interact with, sharing specific real-world cases that are meaningful to them (Ahn, Butler, Alam, \& Webster, 2013).

In a structured online discussion, facilitators play a crucial role in shaping the discussion to meet their goals, making sure all members engage in a focused and deeper conversation one way or another. In an open online discussion, on the other hand, where there is no fixed structure or facilitator, every participant's action is a driving force that impacts the depth and direction of a conversation.

Employing both quantitative and qualitative methods of analysis, we examined the open online discussion forum, specifically looking at the impact of participant actions as facilitative moves to gain a better understanding of the types of actions in the discussion that inspired deeper engagement with the ideas of interest. This will inform course developers and organizers about the types of actions they should support in order to promote sustained participant engagement. In this paper, we will first share the analysis of the patterns of conversational pairs based on types of posts, and then we will present examples of discussion threads to highlight the ways in which deeper conversation evolves over time.

\section{About the Course}

The online course "Learning Creative Learning" that we looked at, was the third edition of the course started in 2013 (Schmidt et al., 2014), aimed to introduce the constructionist pedagogical approach Creative Learning (Resnick, 2018) to educators around the world. The course was organized in six modules, each designed to take one week to go through. Every module consisted of a short video introducing the topic of the week, short reading materials, a hands-on activity that they can do on their own, reflection prompts to be discussed in the discussion forum, and additional resources for further reading (Figure 1). Each module was independent allowing learners to go through the materials at their own pace, in the order they want. At the same time, participants were encouraged to participate in conversations with other participants in the discussion forum (Figure 2) as well as in the weekly conference call which was an opportunity for participants to engage in synchronous conversations with other participants. For the discussion forum, an open source tool called discourse.org was used. For the weekly conference call, another open source tool called Unhangout (Sethi, McConachie, DeTar \& Schmidt, 2014) was used. The course was designed to 
support learner agency and self-directed learning, rather than driving them with extrinsic rewards such as grades and credentials.

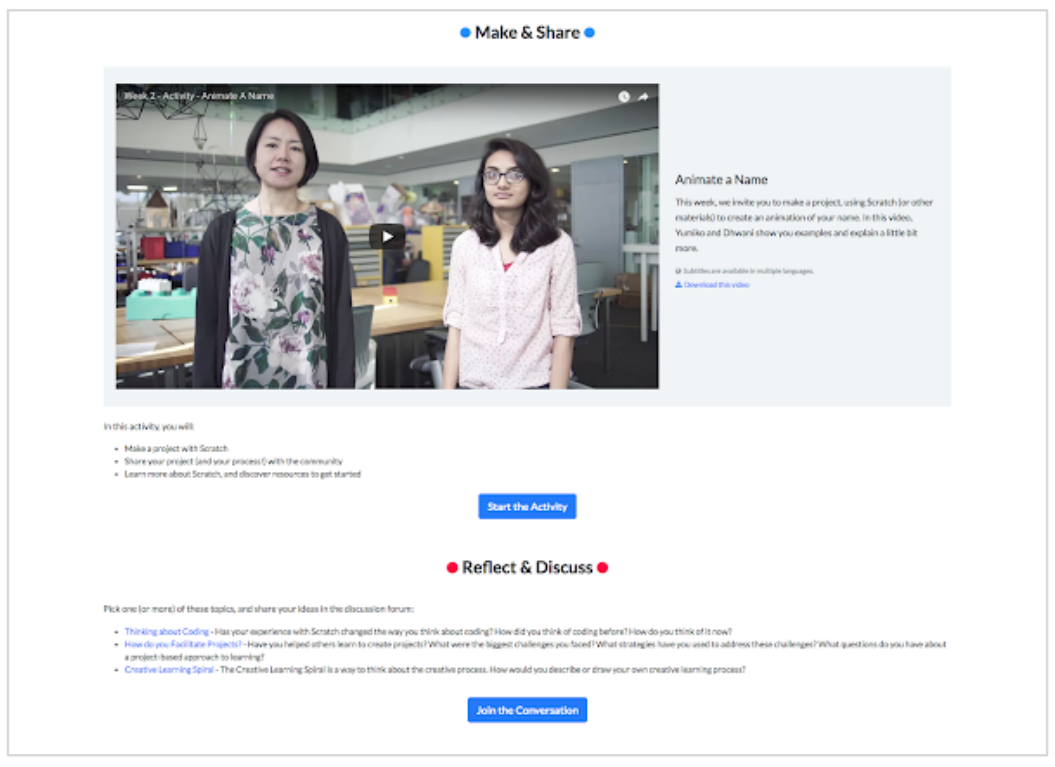

Figure 1: Website

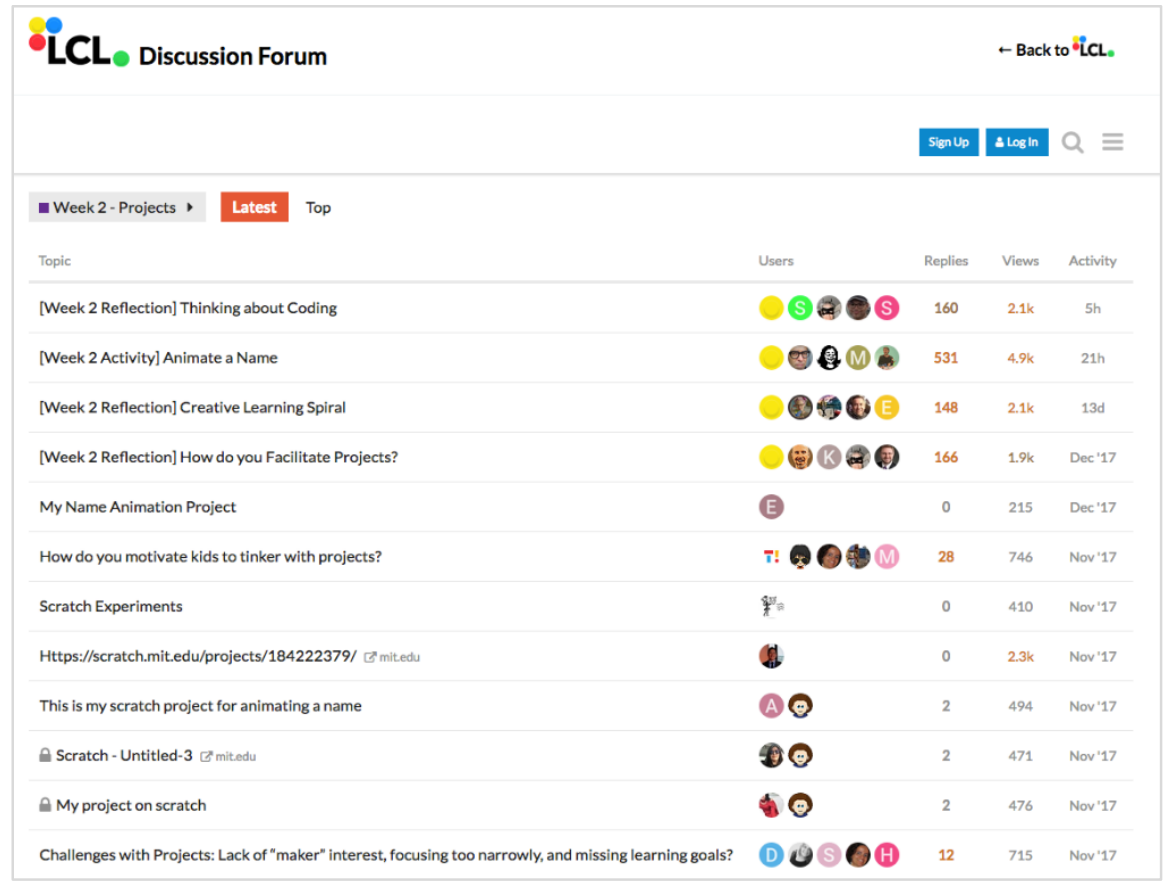

Figure 2: Discussion forum

\section{Methods}

We analysed 32 threads with 172 total posts from the open discussion forum. We started with process coding (Saldana, 2016). The codes were then grouped in smaller number of categories to allow analysis of sequences based on Jeong's (2003) 
Discussion Analysis Tool (DAT). DAT is a predictive tool, created in MS Excel, that estimates which types of sequences of posts or interactions are most likely to occur in a threaded discussion; for example, how likely does a post sharing experience elicit responses as opposed to a post citing a reading; what kind of responses will these posts elicit?

We zeroed down on four categories from the second coding process:

Type 1 (T1). Asking question: Posts that include a question or request to others. Type 2 (T2). Sharing resources: Posts that point readers to an external content such as a paragraph from readings, relevant websites or pedagogical resources. Type 3 (T3). Sharing opinion, practice, experience or anecdote: Posts that are primarily about the participant's own ideas or experiences.

Type 4 (T4). Acknowledging: Posts that are intend to thank the respondent, applauding or what Lowes (2007) terms cheerleading.

Very few posts were purely of one type. Therefore, we also considered combinations of categories i.e. $\mathrm{T} 1+2, \mathrm{~T} 1+3$, and $\mathrm{T} 2+3$ bringing us to seven categories. DAT was used to build matrix of sequences (post type-response type) and calculate probability for each type following the other.

The sequential analysis was chosen to explore the type of posts that keeps conversations going. In contrast to content analysis, it focuses not on the individual message, but on the relationship between messages in a threaded discussion. The DAT tool helped handle multiple levels of branching sub-threads instead of manually counting permutations and combinations of interaction units. We also conducted a close reading of two conversation threads to see if/in what way multi-level threaded discussions that seem robust structurally, build on each other's ideas. The threads selected for close reading had similar sequence structures and had multiple levels of sub-threads.

\section{Findings and Discussion}

The sequence analysis revealed that out of 172 posts 113 posts shared personal experiences or opinions (T3) either by themselves or in combination with a question (T1) based on it. Here are some key points we gleaned from the probability matrix:

- A reply just acknowledging the previous post did not get any response. It ended the conversation.

- If a post shared only a resource (T2) there was a $40 \%$ chance that it received just acknowledgement in response and the conversation died after that. If there was a question embedded in the post, the reply rate was $87 \%$ and the responses were mostly of the type "sharing experience, suggesting practice, sharing opinion"

- There was about $60 \%$ or more chance that responses focused on sharing experiences or opinions about teaching practices (T3), when the post was mainly personal experience (T3), a question (T1), or a question in combination with other categories. Participants seemed to be more comfortable sharing tangible things such as teaching experiences or 'how to' of practice rather than discussing abstract ideas/concepts. 
These results highlight the importance of sharing personal experiences and opinions in attracting more contributions from other participants. In order to further understand how dialogues evolved and to triangulate the findings from the matrix analysis, we took a closer look at two threads. Both threads consisted of multiple lines of conversation that were structurally complex.

Thread A was an example in which the original proposer showed a high degree of commitment to facilitate responses posted in the thread. The original proposer replied to most responses, either acknowledging the contribution or sharing further related experiences. Interestingly, while the original proposer's responsiveness kept the thread active, there was almost no follow-up response when she acknowledged replies. This is consistent with our matrix analysis described above. Social presence of facilitators is deemed important for encouraging participation in an online forum (Garrison, Anderson \& Archer, 2000). However, the frequent response by this original proposer did not necessarily inspire others to make more contribution, and as a result, the responses did not build on each other's ideas.

Another pattern that stood out in this thread was that the sub-threads that slightly diverged from the original post tended to prosper more than the ones that were strictly answering the original question. This may imply that both acknowledgement and the response specifically pointing to the question closed down the conversation by signifying that the interaction with the original poster had concluded.

In contrast to Thread A, Thread B was started with a conceptual question about creative learning, the pedagogical approach featured in this course. Although some responses engaged with the concept itself exploring its nuances, it was difficult to keep participants focused on the abstract. Many of the participants responded with a post exploring the nuances of the abstract concept by sharing what it meant for their practice. As a result, wherever the sub-threads started, it ended in sharing experiences and inspired responses comparing and contrasting each other's experiences.

In contrast to the weekly discussion prompts, the open discussion space allowed participants to share personal stories in relation to the questions they had. It also enabled people to collectively explore an idea, when there was no correct answer. By exchanging their questions and their thoughts back and forth, participants built a deeper understanding of the topic. These observations point to the value of having open discussion spaces for participants in online learning communities.

\section{Conclusion}

This study examined an open online discussion forum to explore how different types of participant actions in the discussion inspired or hindered engagement with the idea of interest.

Overall, the analysis highlighted the value of having an open discussion space in an online course. Open discussion space not only allowed people to collectively build understanding about a topic but also helped them ground those discussion in personal experiences. We also found that a post acknowledging the previous post, thanking for 
resources provided, or agreeing with the previous post might be useful to build community, but by itself, it made it difficult for the conversations to last. On the other hand, an interaction perceived as incomplete or a diverging response, tended to create a space for others to contribute more.

The purpose of the study was to hone the course design and delivery. We identified the following implications for design and facilitation. Firstly, the course organizers can model posting open-ended questions or responses. For example, they can post a response highlighting facets of the original post that are not yet discussed but has a personal connection. Secondly, they can encourage participants to be responsive, but not for the sake of responding. The analysis also implied that sometimes deviating from conversation ends up in a more engaging interaction. This means that rather than just leaving a thank-you note, adding a question, a piece of resource, or pointing to a different way of looking at a concept might help others to join the conversation. Thirdly, it is important to create a culture where people feel comfortable to share their personal experiences. The facilitators can model this type of participation by sharing her own experiences.

In addition to the design implications, this also proved to be an exploration of the ways to look at large scale discussion forum data with a combination of quantitative and qualitative analysis that can be applied to other medium scale discussion forums. 


\section{References}

Ahn, J., Butler, B. S., Alam, A., \& Webster, S. A. (2013). Learner participation and engagement in open online courses: Insights from the Peer 2 Peer University. Journal of Online Learning and Teaching, 9(2), 160-171.

Cormier, D. (2014). Rhizo14 -The MOOC that community built. International Journal for Innovation and Quality in Learning, 107.

Garrison, D., Anderson T. \& Archer, W (2000). Critical inquiry in a text-based environment: Computer conferencing in higher education. The Internet and Higher Education, 2: 87-105.

Hollands, F. M. \& Tirthali, D. (2014). MOOCs: Expectations and reality. Center for Benefit-Cost Studies of Education, Teachers College, Columbia University, 138.

Jeong, A. (2003). Sequential analysis of group interaction and critical thinking in online threaded discussions. American Journal of Distance Education, 17(1), 25-43.

Lowes, S., Lin, P., \& Wang, Y. (2007). Studying the Effectiveness of the Discussion Forum in Online Professional Development Courses, Journal of Interactive Online Learning, 6 (3). 181-210.

Mackness J. \& Bell F. (2015) Rhizo14: A Rhizomatic Learning cMOOC in Sunlight and in Shade. Open Praxis 7(1), pp. 25-38.

Resnick, M. (2017). Lifelong Kindergarten: Cultivating Creativity through Projects, Passions, Peers, and Play. Cambridge, MA: MIT Press.

Saldana, J. (2016). The Coding Manual for Qualitative Researchers. Thousand Oaks, CA: Sage Publications.

Schmidt, P., Resnick, M., Rusk, N. (2014). Learning Creative Learning: How we tinkered with MOOCs. Retrieved from http://reports.p2pu.org/learning-creativelearning.

Sethi, S., McConachie, K., DeTar, C., \& Schmidt, P. (2014). Unhangout. MIT Media Lab. Retrieved from http://unhangout.media.mit.edu

Swan, K., Day, S., Bogle, L., \& van Prooyen, T. (2015). AMP: A tool for characterizing the pedagogical approaches of MOOCs. MOOCs and open education around the world, 105-118. 